\title{
Características del cuidado familiar a pacientes dependientes en programa de hemodiálisis
}

\author{
Lola Andreu Periz* - Carmen Moreno Arroyo* - Maricel Julve Ibáñez**
}

* Facultat d'Infermeria, Universitat de Barcelona

** Hospital Universitario de Bellvitge. L' Hospitalet de Llobregat. Barcelona

"Este trabajo ha sido subvencionado por el Fondo de Investigación Sanitaria". Proyecto PI08/90011.

\section{Sr. Director:}

La edad de los pacientes incluidos en programa de hemodiálisis es cada vez más elevada y se acompaña de una gran morbilidad, esto hace que muchos presenten un grado de dependencia muy importante. La dependencia puede entenderse como el resultado de un proceso que se inicia con la aparición de un déficit en el funcionamiento corporal como consecuencia de una enfermedad 0 accidente. Este déficit comporta una imitación en la actividad. Cuando esta limitación no puede compensarse mediante la adaptación del entorno, provoca una restricción en la participación que se concreta en la dependencia de la ayuda de otras personas para realizar las actividades de la vida cotidiana. Las necesidades de estas personas abarcan muchos aspectos que implican a la familia y a los servicios sociales, supone unos costos personales y económicos importantes que siempre se han cubierto de forma insuficiente Estos cuidadores que habitualmente son sus familiares directos son fundamentales tanto para facilitar el tratamiento como para ofrecer una calidad de vida adecuada a los pacientes.

Nuestro objetivo fue el de conocer las principales características sociodemográficas de pacientes con falta de autonomía personal sometidos a hemodiálisis y de sus cuidadores familiares y conocer

Correspondencia:

L. Andreu: Iolaandreu@ub.edu el impacto que causa el proceso de cuidar en los cuidadores de los pacientes que han participado en el estudio

Se trata de un estudio descriptivo transversal en una muestra de 433 pacientes en programa de hemodiálisis y sus cuidadores familiares. Las variables estudiadas en el grupo de cuidadores fueron sexo, edad, relación familiar, situación laboral y percepción de la propia salud. En el grupo de pacientes se analizó la edad, el sexo y el grado de dependencia de acuerdo con el baremo definido por la Ley sobre promoción de la Autonomía personal y Atención a las personas en situación de dependencia, que establece tres niveles de dependencia (moderado, severo y gran dependencia). Se analizaron también otras variables relacionadas con cuidados que tenía que asumir el cuidador durante el periodo interdiálisis como la administración de medicación o la vigilancia del acceso vascular.

Los resultados obtenidos indican que:

a) Respecto a los pacientes predomina el sexo masculino (69 frente a $61 \mathrm{p}=0,034$ ), siendo la media del tiempo en diálisis de 46 meses (rango 6-112 meses); eran portadores de fístula arteriovenosa interna 198 y 235 se dializaban mediante algún tipo de catéter. El grado de dependencia se relacionó con la edad $(p=0,18)$ y el tiempo en diálisis $(p=0,77)$ Todos los pacientes tomaban durante el periodo interdiálsis más de cuatro fármacos, cuya toma debía ser controlada (ayunas, horario etc.) Los niveles 
de dependencia relacionados con la edad se muestran en la tabla 1.

\begin{tabular}{|l|c|c|c|}
\hline Edad & Gradol & Grado 2 & Grado 3 \\
\hline $0-39$ & 12 & 2 & 1 \\
\hline $40-59$ & 57 & 35 & 10 \\
\hline $60-65$ & 75 & 41 & 9 \\
\hline $70-79$ & 59 & 55 & 4 \\
\hline Más de 80 & 37 & 34 & 2 \\
\hline
\end{tabular}

Tabla 1. Niveles de dependencia y edad de los pacientes

b) Respecto a los cuidadores el género es mayoritariamente femenino (77\%). Los hombres cuidadores tiene una edad media de 76 años (rango 45-84 y las mujeres 68 (rango 38-86). Respecto a la relación familiar, 252 cuidan de su pareja, 120 cuidan a su padre o madre, 20 cuidan de un hijo y 39 de otro familiar o amigo. De todos los cuidadores 46 trabajaban fuera de casa a jornada completa y 380 recibían algún tipo de ayuda en las tareas domésticas. Respecto a la percepción que de la propia salud tiene el cuidador; 294 dicen tenerla buena, regular 144 y mala 59. De todos los cuidadores 232 dicen haber recibido una formación específica para el cuidado del paciente respecto a la hemodiálisis. De todos los cuidadores, cuando tiene dudas respecto a los cuidados que deben proporcionar en el domicilio, acuden a el/la enfermera/o de diálisis (72\%), al nefrólogo/a (12\%), al personal sanitario del Centro de Salud (8\%) y a otros el $10 \%$. El análisis de los datos muestra que el cuidador familiar tipo es una mujer por debajo de los 70 años que no trabaja fuera de casa y que cuida a un familiar de primer grado.

Estos resultados permiten afirmar que los pacientes dependientes en programa de hemodiálisis necesitan de los cuidados informales para favorecer la eficacia del tratamiento dialítico y mantener su calidad de vida por lo que sus cuidadores deben estar adecuadamente asesorados y recibir soporte emocional ya que un porcentaje importante aunque tenga ayuda para la realización de las tareas domesticas tiene una percepción negativa sobre su propio estado de salud .

\section{Bibliografía}

1. IMSERS0. Situación y evolución del apoyo informal a los mayores en España. Informe de resultados. Madrid: IMSERS0, 2004: (acceso 10-1-2011). Disponible en:http://www.imsersomayores.csic.es/documentacion/documentos/registro.jsp?id=1002.

2. Casado D, López, G. Vellesa, dependencia i atencions de llarga durada, situació actual i perspectivas de futur. Barcelona. Fundació la Caixa.2008.

3. Ministerio de Trabajo y Asuntos Sociales. Libro Blanco sobre la atención a las personas en situación de dependencia en España.2004 (acceso 10-1-2011). Disponible en: http:// www.europart.europa.eu/pv2.

4 Andreu L, Moreno C. Perfil y realidad social de los cuidadores principales de pacientes dependientes tratados con hemodiálisis. Nursing 2009 27(4): 54-65. 